\title{
Sistemas agroflorestais no Brasil: uma abordagem sobre a estocagem de carbono
}

Carlos Moreira Miquelino Eleto Torres ${ }^{1}$, Laércio Antônio Gonçalves Jacovine ${ }^{1}$, Silvio Nolasco de Oliveira Neto ${ }^{1}$, Daniel Brianezi ${ }^{1}$, Eliana Boaventura Bernardes Moura Alves ${ }^{1}$

${ }^{1}$ Universidade Federal de Viçosa, Departamento de Engenharia Florestal, Campus Universitario, s/nº, CEP 36571-000, Viçosa, MG, Brasil

"Autor correspondente:

carlos.eleto@yahoo.com.br

Termos para indexação:

Sequestro de carbono

Integração lavoura-pecuária-floresta

Sistemas de uso do solo

Index terms:

Carbon sequestration

Crop-livestock-forest integration

Land use

Histórico do artigo:

Recebido em 22/11/2013

Aprovado em 19/06/2014

Publicado em 10/10/2014
Resumo - Os sistemas agroflorestais (SAFs) fazem parte das ações estratégicas de mitigação das emissões de gases de efeito estufa (GEE), que compões a Política Nacional sobre Mudanças do Clima (PNMC). Considerando a importância destes sistemas na captura e estocagem de carbono atmosférico, é preciso avaliar esse potencial de armazenagem de carbono, a fim de incentivar essa atividade no contexto das políticas públicas brasileira sobre mudanças climáticas. Neste sentido, objetivou-se sistematizar os dados disponíveis no acervo bibliográfico que demonstrem a estocagem de carbono de diferentes arranjos agroflorestais. Os dados indicaram uma grande variação no incremento médio de carbono nos SAFs, variando de $1,26 \mathrm{t} \mathrm{C} \mathrm{ha}^{-1}$ ano $^{-1}$ a $11,19 \mathrm{t} \mathrm{C} \mathrm{ha}^{-1}$ $\mathrm{ano}^{-1}$, dependendo da sua estrutura e idade. Sendo assim, as estimativas de estocagem de carbono nos SAFs devem ser um dos fatores a serem considerados para incentivar essa atividade e na escolha do arranjo a ser adotado. Por fim, infere-se que esses sistemas são de grande importância para tornar práticas agropecuárias ambientalmente mais sustentáveis e para a mitigação de emissão de GEE.

\section{Agroforestry systems in Brazil: an approach about carbon storage}

\begin{abstract}
The agroforestry systems (AFS) are part of the strategic actions to mitigate emissions of greenhouse gases (GHG), which compose the National Policy on Climate Change (NPCC). Considering the importance of these systems in capturing and storing atmospheric carbon it is necessary to evaluate this potential of carbon storage in order to encourage this activity in the context of Brazilian public policy on climate change. In this sense, it is aimed to systematize the available data in the bibliographic, demonstrating carbon storage of different agroforestry arrangements. The data indicated large variation in the average increment of carbon in agroforestry systems ranging from $1.26 \mathrm{t} \mathrm{C} \mathrm{ha}^{-1} \mathrm{yr}^{-1}$ to $11.19 \mathrm{t} \mathrm{C} \mathrm{ha}^{-1} \mathrm{yr}^{-1}$, depending on its structure and age. Thus, estimates of carbon storage in AFS should be one of the factors to be considered to encourage this activity and the choice of arrangement to be adopted. Finally, we infer that these systems have a great importance to make agricultural management practices more environmentally sustainable and for the mitigation of GHG emissions.
\end{abstract}




\section{Introdução}

O Brasil, em 2009, durante a $15^{\text {a }}$ Conferência das Partes (COP-15), ocorrida em Copenhague, Dinamarca, assumiu reduzir de forma voluntária as emissões de gases de efeito estufa (GEE), entre $36,1 \%$ e $38,9 \%$, dos níveis de 2005, até 2020. Como parte integrante desta política existe a necessidade de identificar e quantificar as principais fontes de emissões e remoções de GEE, por meio de inventários nacionais e corporativos. Além disso, como o Brasil é signatário da Convenção Quadro das Nações Unidas sobre Mudança do Clima (CQNUMC), tem como obrigação a elaboração e atualização periódica do inventário nacional de emissões antrópicas por fontes e remoções por sumidouros de gases de efeito estufa não controlados pelo protocolo de Montreal (Brasil, 2010).

Esse inventário relata que no Brasil, diferentemente dos países industrializados, a principal fonte das emissões líquidas estimadas de dióxido de carbono $\left(\mathrm{CO}_{2}\right)$ é proveniente da mudança do uso da terra, em particular da conversão de florestas para uso agropecuário, com 77\% dessas emissões, em 2005. Além disso, as atividades agropecuárias em si são as principais fontes de metano $\left(\mathrm{CH}_{4}\right)$ e óxido nitroso $\left(\mathrm{N}_{2} \mathrm{O}\right)$. A fermentação entérica nos ruminantes é a fonte de maior emissão de $\mathrm{CH}_{4}$ no país, com 63,2\%, em 2005. Já para o $\mathrm{NO}_{2}$, os dejetos de animais em pastagem são as mais importantes, com 47,5\% da emissão desse gás (Brasil, 2010).

Para mitigar as emissões de GEE advindas da mudança do uso da terra e cumprir as metas de redução estabelecidas pelo governo federal, criou-se o programa Agricultura de Baixo Carbono (ABC), que tem como objetivo incentivar a adoção de práticas sustentáveis que garantem a redução de emissões de GEE, aliadas ao aumento de renda dos produtores, sobretudo com a expansão das seguintes tecnologias: recuperação de pastagens degradadas; integração lavoura-pecuáriafloresta (iLPF) e sistemas agroflorestais (SAFs); sistema de plantio direto (SPD); fixação biológica de nitrogênio (FBN); e florestas plantadas (Brasil, 2011).

Dessas tecnologias, os sistemas agroflorestais, que envolvem a utilização deliberada de árvores ou outras plantas perenes lenhosas, com cultivos agrícolas, pastagens e/ou animais, visando benefícios das interações ecológicas e econômicas resultantes (Nair, 1993), integram a produção alimentar com fontes de sumidouros do gás carbônico atmosférico $\left(\mathrm{CO}_{2}\right)$, além de consistir em estratégia para a redução de desmatamentos em países tropicais, como o Brasil (Oelbermann et al., 2004).

Além de compor as estratégias das políticas públicas brasileiras, os sistemas agroflorestais são contemplados no mercado de carbono global, dentro do mecanismo de desenvolvimento limpo (MDL). Para que projetos de sistemas agroflorestais gerem as reduções certificadas de emissões, consideradas créditos de carbono do MDL, existem três metodologias para a realização dos projetos. Uma destas, utilizada em larga escala, é o florestamento ou reflorestamento em terras degradadas que permitam atividades silvipastoris (AR-AM0009 - Afforestation or reforestation on degraded land allowing for silvopastoral activities). Outras duas metodologias se referem a projetos de pequena escala: 1) Metodologia simplificada de linha de base e monitoramento para projeto de pequena escala em Sistemas Agroflorestais - atividades de projetos de florestamento e reflorestamento no âmbito do MDL (AR-AMS0004 - Simplified baseline and monitoring methodology for small-scale agroforestry afforestation and reforestation project activities under the clean development mechanism); e 2) Metodologia simplificada de linha de base e monitoramento para projeto de pequena escala em Sistemas Silvipastoris atividades de projetos de florestamento e reflorestamento no âmbito do MDL (AR-AMS0006 - Simplified baseline and monitoring methodology for small-scale silvopastoral - afforestation and reforestation project activities under the clean development mechanism).

Dada a importância dos SAFs na minimização das mudanças climáticas, é preciso conhecer o potencial de estocagem de carbono nesses sistemas, de forma a balizar políticas públicas de incentivo às atividades. Neste sentido, objetivou-se analisar a estocagem de carbono em diferentes modelos de Sistemas Agroflorestais.

\section{Caracterização do estudo}

Este trabalho foi desenvolvido por meio de análise e discussão de publicações científicas publicadas sobre a estocagem de carbono em diferentes sistemas agroflorestais no Brasil.

A estocagem de carbono foi subdividida de acordo com os compartimentos estabelecidos por Pennam et al. (2003) (Tabela 1). 
Tabela 1. Definição dos sumidouros de carbono terrestre.

\begin{tabular}{|c|c|c|}
\hline \multicolumn{2}{|c|}{ Reservatório } & Descrição \\
\hline \multirow{2}{*}{ Biomassa viva } & $\begin{array}{l}\text { Biomassa acima do solo } \\
\text { (AGB) }\end{array}$ & Biomassa viva acima do solo, incluindo tronco, galhos, cascas, sementes e folhagem. \\
\hline & $\begin{array}{l}\text { Biomassa abaixo do solo } \\
\text { (BGB) }\end{array}$ & Biomassa viva de raízes. \\
\hline \multirow[b]{2}{*}{$\begin{array}{l}\text { Matéria } \\
\text { orgânica } \\
\text { morta }\end{array}$} & Madeira morta & Biomassa lenhosa não viva encontrada no solo e na superfície, excluindo a serapilheira. \\
\hline & Serapilheira & $\begin{array}{l}\text { Biomassa morta com diâmetro inferior ao mínimo estabelecido pelo país (por exemplo, } \\
10 \mathrm{~cm} \text { ), sobre o solo mineral ou orgânico,composta pela serapilheira, camadas fúlvicas } \\
\text { e húmicas. }\end{array}$ \\
\hline Solos & Matéria orgânica no solo & $\begin{array}{l}\text { Carbono orgânico em solos minerais e orgânicos (incluindo turfa) a uma profundidade } \\
\text { específica estabelecida pelo país e monitorada por meio de uma série histórica. }\end{array}$ \\
\hline
\end{tabular}

Fonte: Pennam et al. (2003)

A literatura disponível apresentava dados de estoque de carbono acima do solo e a idade de implantação dos sistemas. Assim, para padronização dos resultados, os dados de estoque foram transformados em incremento médio de carbono acima do solo (IM-AGB). Esse é um método simples, que permite a comparação de resultados, porém deve-se considerar que existem muitas variáveis intervenientes na fixação do carbono ao longo do ciclo dos SAFs, como características de solo, luminosidade, tipo de manejo, etc.

\section{Descrição dos sistemas agroflorestais estudados 1. Sistemas agrossilviculturais}

São caracterizados pela associação de árvores com culturas agrícolas e podem incluir diferentes tipos de consórcios, como sistema agroflorestal regenerativo análogo, culturas em aleias, sistemas agroflorestais com árvores e culturas perenes, entre outros (Nair, 1985).

\subsection{Sistema agroflorestal regenerativo análogo (SAFRA) ou agrofloresta}

O modelo denominado SAFRA caracteriza-se por um sistema de multi-estratos, que busca reproduzir a arquitetura do ecossistema tropical original, onde se aproveita o espaço horizontal e vertical, aumentando a diversidade e otimizando a radiação, umidade e nutrientes, o que possibilita explorar da melhor forma os diferentes estratos que compõe o sistema (Götsch, 1995; Dubois et al., 1996; Vivan, 1998).

Cada SAFRA possui uma dinâmica fotossintética devido às condições edafoclimáticas e pela composição e arranjo espacial das espécies utilizadas, formando assim, classes distintas de estoque de carbono (Winrock International, 1997; Bolfe et al., 2009).

\subsection{Culturas em aleias}

O sistema de cultivo em aleias é um tipo de sistema agroflorestal simultâneo, que consiste na associação de culturas agrícolas intercaladas com árvores e/ou arbustos, geralmente fixadores de nitrogênio. As podas periódicas realizadas nas árvores ou arbustos permitem a melhoria das características químicas do solo, podendo, assim, aumentar sua fertilidade (Mafra et al., 1998; Bertalot, 2003)

Montagnini \& Nair (2004) relatam que os cultivos em aléias podem manter os estoques de carbono do solo pelo ingresso das podas de árvores e pelos restos culturais, além do estoque nos componentes arbóreos, contribuindo com as questões climáticas globais.

\subsection{Sistemas agroflorestais com árvores e culturas perenes}

Neste sistema, árvores, madeireiras e/ ou frutíferas, de sombra são combinadas com culturas perenes tolerantes à sombra, como café, cacau, cupuaçu, banana, coco, chá preto, erva-mate (Engel, 1999). As culturas perenes apresentam vantagens, pois, além de estocar o carbono por um período de tempo maior, a sua exploração não necessariamente acaba com o corte da madeira, o que poderia levar à emissão de GEE (Albrecht \& Kandji, 2003)

\section{Sistemas com cacau}

O cacau (Theobroma cacao) é uma espécie ombrófila, oriunda do sub-bosque da Floresta Amazônica, que foi tradicionalmente cultivado em SAFs, principalmente no sudeste do Estado da Bahia (Piasentin \& Saito, 2012).

O cacau tem sido plantado em sub-bosques de florestas nativas, sistema cabruca, ou consorciado com espécies florestais, que apresentam um longo período de maturidade e são plantadas em espaçamentos amplos, como a seringueira (Hevea brasiliensis). O sistema cabruca é um sistema tradicional de cultivo do cacau no sul da Bahia, baseado na ocupação do sub-bosque por uma cultura de interesse econômico, geralmente o 
cacau, implantada de forma descontínua e circundada por vegetação natural, não afetando as relações com os sistemas remanescentes (Lobão et al., 2004).

Sambuichi (2006), estudando um sistema cabruca no Município de Ilhéus, BA, afirma que os altos valores de área basal e de incremento diamétrico encontrados em seu estudo, são indicativos de que as cabrucas podem ser utilizadas para o sequestro e estoque de carbono.

\section{Café sombreado}

O café é uma cultura que está sofrendo consequências, como a perda de produtividade, devido às mudanças climáticas globais. Deconto (2008) relata que com tais mudanças a cultura poderá sofrer por deficiência hídrica ou por excesso térmico nas regiões tradicionais. Estados como São Paulo e Minas Gerais poderão perder boa parte da área hoje cultivada, e regiões do Paraná, Santa Catarina e Rio Grande do Sul poderão ter aumento na produção, porém não suficiente para compensar as perdas gerais da cultura na agricultura brasileira.

Uma medida para mitigação das mudanças climáticas e dos impactos gerados por esta para a cultura do café é a arborização dos cafezais. As árvores podem trazer benefícios para a cultura, como a redução da temperatura do solo e do ar, da velocidade do vento e o aumento da umidade relativa (UR) (DaMatta, 2004), minimizando possíveis perdas provenientes das mudanças climáticas, além de serem sumidouros do $\mathrm{CO}_{2}$ atmosférico.

\section{Sistemas silvipastoris e agrossilvipastoris e a estocagem de carbono}

Sistemas silvipastoris são formas de uso da terra nos quais árvores ou arbustos são combinados com a produção de pastagem e animais, em uma mesma unidade de área. Já nos sistemas agrossilvipastoris, além das árvores e pastagem/animais, existe a associação desses elementos com culturas agrícolas (Nair, 1993).

Esses sistemas são muito importantes para a mitigação e/ou compensação dos GEE emitidos pelo setor agropecuário brasileiro (Nair et al., 2011). Segundo Brasil (2010), a pecuária, por meio da fermentação entérica dos ruminantes, é a maior fonte de emissão de $\mathrm{CH}_{4}$, com 63,2\%, em 2005, além de emitir mais $\mathrm{CH}_{4} \mathrm{e}$ $\mathrm{N}_{2} \mathrm{O}$, por meio do manejo de dejetos dos animais. Com isso, a arborização de pastagens pode contribuir para a captura de carbono e para a menor emissão de $\mathrm{N}_{2} \mathrm{O}$, devido à melhoria de qualidade de vida dos animais, e para a mitigação da emissão de $\mathrm{CH}_{4}$ pelos ruminantes (Porfírio-da-Silva, 2006).

\section{Resultados}

Os resultados de estocagem de carbono dos diferentes arranjos agroflorestais são apresentados na Tabela 2.

\section{Estocagem de carbono em sistemas agrossilviculturais \\ Sistema agroflorestal regenerativo análogo (SAFRA) ou agrofloresta}

Em estudo com diferentes modelos de sistemas agroflorestais - SAF-1, composto por cacau (Theobroma cacao L.), açaí (Euterpe oleracea Mart.), bananeira (Musa cavendishii L.) e seringueira (Hevea brasiliensis Muell. Arg.), aos 14 anos de idade; SAF-2, composto por cacau, açaí, bananeira, seringueira, taperebá (Spondias mombin L.), paricá (Schizolobium amazonicum Huber ex Ducke) e macaúba (Platymiscium trinitatis Benth.), aos 14 anos; SAF-3, composto por cupuaçu (Theobroma grandiflorum L.), açaí, teca (Tectona grandis Lf) e mogno (Swietenia macrophylla King), aos 9 anos; e SAF-4, composto por cupuaçu, açaí e paricá, aos 9 anos, no Município de Tomé-Açu, PA, observou-se um estoque de carbono total (biomassa aérea, serapilheira e solo) de $126,59 \mathrm{t} \mathrm{C} \mathrm{ha}^{-1} ; 128,41$ t C ha- ${ }^{-1} ; 122,80$ t C ha $^{-1}$ e 137,81 t C ha $^{-1}$, para os SAFs $1,2,3$ e 4, respectivamente. Assim, o incremento médio de carbono acima do solo (IM-AGB) dos sistemas correspondem a 2,86 t $\mathrm{C} \mathrm{ha}^{-1} ; 3,15 \mathrm{tC} \mathrm{ha}^{-1} ; 4,14 \mathrm{tC} \mathrm{ha}^{-1} \mathrm{e}$ $4,88 \mathrm{tC} \mathrm{ha}^{-1}$ ano $^{-1}$, para os SAFs $1,2,3 \mathrm{e} 4$, respectivamente (Brancher, 2010).

Outro estudo envolvendo SAFRA, formado a partir do manejo de uma floresta nativa já explorada, com elevada densidade de palmeiras (principalmente açaí) e frutíferas (principalmente cacau), aos 12 anos, foi realizado nas várzeas do rio Juba, Cametá, PA. Para estimar a biomassa e carbono da parte áerea (madeira/estipe e folhas) dos SAFs, a vegetação foi dividida em açaí, cacau e árvores (espécies nativas pré-existentes). O estoque médio total de carbono da vegetação foi de $134,30 \mathrm{t} \mathrm{ha}^{-1}$; no qual 131,63 t ha-1 $(98 \%)$ foram estocadas nas árvores, $2,01 \mathrm{t} \mathrm{ha}^{-1}(1,5 \%)$ no açaí e $0,65 \mathrm{t} \mathrm{ha}^{-1}(0,5 \%)$ no cacau. O IM-AGB encontrado foi de $11,19 \mathrm{t} \mathrm{ha}^{-1}$ ano $^{-1}$ (Santos et al., 2004).

Froufe et al. (2011) avaliaram o estoque de carbono de agroflorestas compostas por espécies agrícolas (com predominância de banana) e espécies florestais, incluindo nativas e exóticas; capoeiras em diferentes estágios de regeneração, sistemas agrícolas convencionais e pastagens, na região do Alto Vale do Ribeira, SP. 


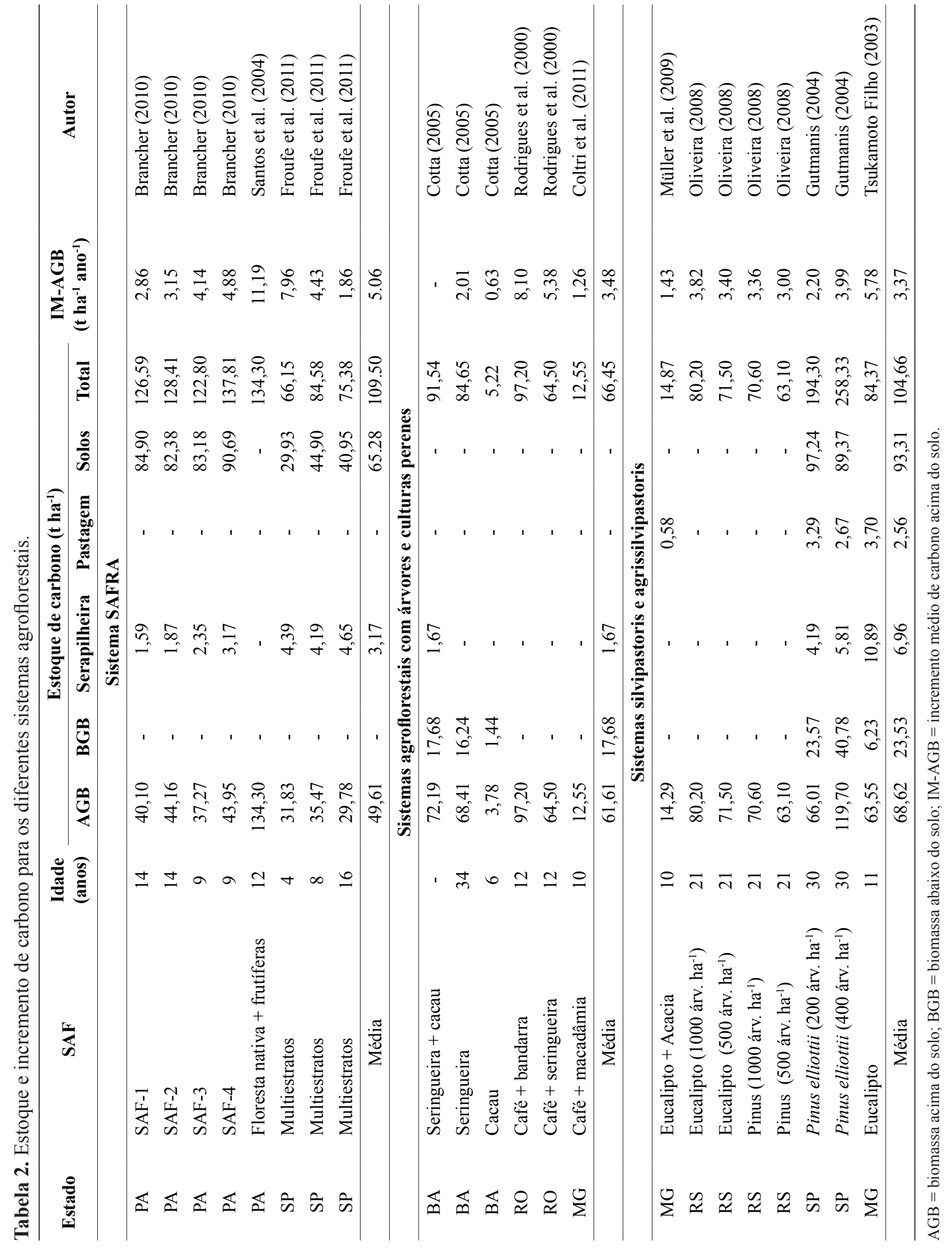


Os autores observaram que, em média, as capoeiras apresentaram maior estoque de carbono, 115,78 $\mathrm{t} \mathrm{ha}{ }^{-1}$, seguidas por agroflorestas com $75,37 \mathrm{t} \mathrm{ha}^{-1}$, cultivos agrícolas com 47,07 $\mathrm{t} \mathrm{ha}^{-1} \mathrm{e}$ das pastagens com 36,01 $\mathrm{t} \mathrm{ha}^{-1}$. O IM-AGB dos SAFs variou entre $1,86 \mathrm{t} \mathrm{C} \mathrm{ha}^{-1} \mathrm{ano}^{-1}$ e $7.96 \mathrm{t} \mathrm{C} \mathrm{ha}^{-1} \mathrm{ano}^{-1}$. O SAF com 4 anos obteve uma estocagem de carbono total (biomassa acima do solo + serapilheira + solo) de 66,15 t ha ${ }^{-1}$ e um IM-AGB de $7.96 \mathrm{t} \mathrm{ha}^{-1} \mathrm{ano}^{-1}$. O SAF com 8 anos obteve estocagem de 84,58 tha $^{-1} \mathrm{e}$ incremento de 4,43 $\mathrm{t} \mathrm{ha}^{-1} \mathrm{ano}^{-1}$ e o de 16 anos obteve $75,38 \mathrm{tha}^{-1} \mathrm{e} 1,86 \mathrm{tha}^{-1}$ $\mathrm{ano}^{-1}$, para a estocagem e incremento, respectivamente.

\section{Estoque de carbono nas culturas em aléias}

Em um sistema em aléias, com leucena (Leucaena leucocephala (Lam.) de Wit.) e com o cultivo intercalar de centeio (Secale cereale L.) + aveia (Avena ssp.) e milho (Zea mays L.) + feijão (Phaseolus vulgaris L.), no Município de Botucatu, SP, Mafra et al. (1998) encontraram uma produção anual de biomassa de $11,04 \mathrm{tha}^{-1} \mathrm{ano}^{-1}$, sendo que a poda da leucena produziu 4,47 tha ${ }^{-1}$ ano $^{-1}$, a serapilheira 2,40 t ha $^{-1}$ ano $^{-1}$ e os restos culturais, que estavam presente na serapilheira, $4,17 \mathrm{t} \mathrm{ha}^{-1}$ ano $^{-1}$.

Sistemas agroflorestais com árvores e culturas

\section{perenes}

Sistemas com cacau

Em um sistema com seringueira e cacau, no Município de Igrapiúna, BA, composto por seringueiras com 34 anos de idade, e cacaueiros com 6 anos, verificou-se 91,54 t $\mathrm{C} \mathrm{ha}^{-1}$ de estoque de carbono, sendo que 84,65 tC ha ${ }^{-1}$ estavam estocados nas seringueiras $\left(68,41 \mathrm{tC} \mathrm{ha}^{-1}\right.$ na parte aérea e 16,24 t C ha-1 nas raízes), 5,22 t C ha-1 nos cacaueiros $\left(3,78 \mathrm{tC} \mathrm{ha}^{-1}\right.$ na parte aérea e $1,44 \mathrm{tC} \mathrm{ha}^{-1}$ nas raízes) e $1,67 \mathrm{t} \mathrm{C} \mathrm{ha}^{-1}$ na serapilheira. A seringueira apresentou um IM-AGB de 2,01 t C ha-1 ano $^{-1}$ e o cacau 0,63 t C ha ${ }^{-1}$ ano $^{-1}$ (Cotta, 2005).

\section{Café sombreado}

Em sistemas agroflorestais compostos por café e bandarra (Schizolobium amazonicum) e café e seringueira (Hevea brasiliensis), aos 12 anos, nos Municípios de Theobroma e Ji-Paraná, RO, Rodrigues et al. (2000) observaram estoque de C, acima do solo, de 97,2 $\mathrm{tC} \mathrm{ha}^{-1}$ e 64,5 $\mathrm{tC} \mathrm{ha}^{-1}$, respectivamente. Os sistemas obtiveram um IM-AGB de 8,10 t C ha-1 ano-1 e 5,38 t C ha ${ }^{-1}$ ano $^{-1}$, respectivamente.

Coltri et al. (2011), estudando um sistema cafeeiro arborizado com macadâmia (Macadamia ssp.), aos 10 anos, no Município de São Sebastião do Paraíso, na região sul de Minas Gerais, encontraram um estoque de carbono de 12,55 t C ha-1, sendo que as plantas de café estocaram 10,65 t C ha-1, e as de macadâmia $1,9 \mathrm{t} \mathrm{C} \mathrm{ha-1}^{-1}$. O sistema obteve um IM-AGB de 1,26 t C ha-1 ano $^{-1}$.

Sistemas silvipastoris e agrossilvipastoris e a estocagem de carbono

Em um sistema silvipastoril misto, com eucalipto (Eucalyptus grandis) e acácia (Acacia mangium), implantado na Zona da Mata mineira, aos 10 anos, com densidade de 105 árv. ha ${ }^{-1}$, sendo 60 árvores de eucalipto e 45 árvores de acácia, observou-se um estoque estimado de $14,29 \mathrm{tC}$. ha- ${ }^{-1}$, no qual $11,17 \mathrm{tC} \mathrm{ha}^{-1}$ estavam estocados no eucalipto e 3,12 na acácia. O sistema apresentou um IM-AGB de 1,43 t C ha-1 ano-1 (Müller et al., 2009).

Em sistemas silvipastoris com eucalipto (Eucalyptus grandis) e pinus (Pinus elliottii), em diferentes áreas e com densidades de 1.000 e 500 árvores por hectare, no Município de Alegrete, RS, aos 21 anos, o estoque de carbono acima do solo do sistema com pinus foi de 63,1 $\mathrm{tCha}^{-1} \mathrm{e} 70,6 \mathrm{tC} \mathrm{ha}^{-1}$, e o IM-AGB foi de 3,00 tC ha- $\mathrm{e}^{-1} 3,36$ t $\mathrm{C}$ ha $^{-1}$ ano $^{-1}$, para densidades de 500 e 1.000 árvores por hectare, respectivamente. No sistema com eucalipto, o estoque de carbono acima do solo foi de 71,5 t $\mathrm{C} \mathrm{ha}^{-1}$ e 80,2 t C ha ${ }^{-1}$ e o IM-AGB foi de 3,40 $\mathrm{t} \mathrm{C} \mathrm{ha}^{-1}$ e 3,82 t C ha ${ }^{-1}$ ano $^{-1}$, para densidades de 500 e 1.000 árvores por hectare, respectivamente (Oliveira, 2008).

No Município de Nova Odessa, SP, Gutmanis (2004) avaliou duas densidades populacionais de Pinus elliottii (200 e 400 árv. ha ${ }^{-1}$ ) em sistema silvipastoril, aos 30 anos de idade, e observou um estoque de carbono de 89,58 t C ha ${ }^{-1}$ para as árvores $\left(66,01 \mathrm{t} \mathrm{C} \mathrm{ha}^{-1}\right.$ acima do solo e 23,57 $\mathrm{tC} \mathrm{ha}^{-1}$ abaixo do solo), 3,29 $\mathrm{tC} \mathrm{ha}^{-1}$ para o capim, 4,18 $\mathrm{t} \mathrm{C} \mathrm{ha}^{-1}$ para a serapilheira e $97,24 \mathrm{t} \mathrm{C}^{-1}$ no solo. Além disso, apresentou um IM-AGB de 2,20 t C ha-1 ano $^{-1}$, na densidade de 200 árv. ha ${ }^{-1}$. Na densidade de 400 árv.ha ${ }^{-1} \mathrm{o}$ autor observou um estoque de $160,48 \mathrm{t} \mathrm{C} \mathrm{ha}^{-1}$ para as árvores $\left(119,70 \mathrm{t} \mathrm{C} \mathrm{ha}^{-1}\right.$ acima do solo e 40,78 t C ha-1 abaixo do solo), 2,67 t C ha ${ }^{-1}$ para o capim, 5,81 t C ha ${ }^{-1}$ para a serapilheira e $89,37 \mathrm{tC} \mathrm{ha}^{-1}$ no solo. Além disso, apresentou um IM-AGB de 3,99 t C ha- ano $^{-1}$.

Tsukamoto Filho (2003), ao estudar a fixação de carbono em um sistema agrossilvipastoril com eucalipto, no Município de Paracatu, MG, observou um estoque de carbono no sistema de $84,37 \mathrm{t} \mathrm{C} \mathrm{ha}^{-1}$, aos 11 anos, sendo que 63,55 t C ha-1, 6,23 t $\mathrm{C} \mathrm{ha}^{-1}, 10,89 \mathrm{t} \mathrm{C}^{-1} \mathrm{e}^{-1}$ $3,70 \mathrm{t} \mathrm{C} \mathrm{ha}^{-1}$ estavam estocados na biomassa acima do solo, biomassa abaixo do solo, serapilheira e pastagem, respectivamente. O sistema apresentou um IM-AGB de $5,78 \mathrm{t} \mathrm{C} \mathrm{ha}^{-1}$ ano $^{-1}$. 


\section{Discussão}

\section{Biomassa acima do solo}

A biomassa acima do solo geralmente representa a maior fração de biomassa viva total em uma floresta (Brown, 1997). A dinâmica de produção dessa biomassa é alterada de acordo com o desenvolvimento da floresta. À medida que a floresta se desenvolve ocorre uma redução gradual da biomassa da copa das árvores, aumentando a proporção dos componentes madeira e casca. Em uma floresta madura esses componentes representam, em média, mais de $80 \%$ da biomassa acima do solo (Schumacher \& Hoppe, 1997).

Nos sistemas analisados, verificou-se que todos tinham os dados sobre a biomassa estimada acima do solo, pois este é o compartimento de mais fácil mensuração. Os sistemas que obtiveram o maior estoque foi o SAFRA com floresta nativa + frutíferas, aos 12 anos, no Estado do Pará, com uma estocagem de 134,30 t $\mathrm{C} \mathrm{ha}^{-1}$, seguido pelo sistema silvipatoril com Pinus elliottii (400 árv. ha $^{-1}$ ), aos 30 anos, no Estado de São Paulo, com 119,70 $\mathrm{t} \mathrm{C} \mathrm{ha-1}$, e pelo sistema com café + bandarra, aos 12 anos, no Estado de RO, com 97,20 t C ha-1 (Tabela 2).

A estocagem feita pelo sistema com café + bandarra foi similar ao encontrado por Schumacher \& Witschoreck (2004), em um inventário de carbono em povoamentos de eucalipto na Região Sul do Brasil, em que obtiveram um estoque de carbono, aos 8 anos, igual a 97,86 $\mathrm{tha}^{-1}$.

As médias de 49,61 t ha-1; 61,61 $\mathrm{t} \mathrm{ha}^{-1}$; e 68,62 t ha ${ }^{-1}$ para o SAFRA, sistemas agroflorestais com árvores e culturas perenes e sistemas silvipastoris e agrossilvipastoris, respectivamente, foram superiores ao encontrado por Paixão (2004), quando estudou a quantificação do estoque de carbono em um povoamento de eucalipto, aos 6 anos, no Município de Viçosa, MG, que foi de 47,7 t C ha ${ }^{-1}$.

Quando se analisa o IM-AGB verifica-se que existe uma variação de $1,26 \mathrm{t} \mathrm{C} \mathrm{ha}^{-1}$ ano $^{-1}$ a $11,19 \mathrm{t} \mathrm{C} \mathrm{ha}{ }^{-1}$ ano ${ }^{-1}$, dependendo da composição, arranjo e idade dos sistemas. $\mathrm{O}$ modelo denominado sistema agroflorestal regenerativo análogo, em média, foi o que obteve o maior IM-AGB. Isso acontece, pois o SAFRA apresenta um maior número de espécies arbóreas e/ou arbustivas existentes, por unidade de área, favorecendo a estocagem de carbono (Mosquera-Losada et al., 2011).

Os valores encontrados para IM-AGB são compatíveis com os obtidos por Reis et al. (1994), em plantios de eucalipto no Estado do Espírito Santo, que determinaram um IM-AGB de 8,05 tha-1 ano $^{-1}$, correspondendo a $78 \%$ da estocagem total (65\% provenientes da biomassa do tronco e $13 \%$ da copa). Os valores também são similares ao estudo de Mello \& Gonçalves (2008), que avaliaram plantações comerciais de eucalipto (Eucalyptus grandis), aos 9 anos de idade, em dois sítios no planalto ocidental do Estado de São Paulo. Os autores encontraram para o sítio mais produtivo e para o menos produtivo um IM-AGB de 7,25 $\mathrm{t} \mathrm{ha}^{-1} \mathrm{ano}^{-1}$ e 5,20 t ha- $\mathrm{ano}^{-1}$, respectivamente.

\section{Biomassa abaixo do solo}

A biomassa abaixo do solo pode ser dividida em raízes finas e grossas. As raízes finas são responsáveis pela absorção de água e nutrientes (Bowen, 1985) e constituem parte importante na estocagem de carbono, porém, em muitos estudos elas não são mensuradas (Ostonen et al., 2005). As raízes grossas são compostas pelas raízes estruturais com maiores dimensões, que são responsáveis pelo suporte da parte aérea e podem acumular cerca de $30 \%$ da biomassa total nos ecossistemas florestais (Brassard et al., 2011).

A relação de alocação da biomassa relativa entre as raízes e a parte aérea é determinada por fatores bióticos, como a espécie e a idade da floresta ou plantio, e fatores abióticos, como a textura, umidade e disponibilidade de nutrientes do solo (Cairns et al., 1997).

A estocagem de carbono nas raízes dos sistemas estudados variou entre $6,23 \mathrm{t} \mathrm{C} \mathrm{ha}^{-1}$ e $40,78 \mathrm{t} \mathrm{C} \mathrm{ha}^{-1}$. Paixão (2004) verificou uma estocagem 14,71 t C ha-1 pelas raízes em um povoamento de eucalipto, aos 6 anos, no Município de Viçosa, MG.

Para os sistemas agroflorestais com árvores e culturas perenes a estocagem nas raízes correspondeu a 19,67\% da biomassa viva, e para os sistemas silvipastoris e agrissilvipastoris foi de $20,22 \%$. Esses valores são próximos ao encontrado por Reis et al. (1994), em plantios de eucalipto no Estado do Espírito Santo, em que as raízes corresponderam a $22 \%$ da estocagem da biomassa viva.

\section{Serapilheira}

A serapilheira é formada por material vegetal da parte aérea das plantas, como frutos, sementes, flores, galhos, cascas e folhas, em que este último, geralmente, representa a maior fração (Espig et al., 2009). A partir do momento que é depositada sobre o solo, a serapilheira é submetida a um processo de decomposição que é iniciada pela ação da fauna edáfica, que degrada o material para 
que este seja, posteriormente, decomposto pelos microorganismos (Calvi et al., 2009). Ao final desse processo, há a liberação eventual dos nutrientes minerais que compõem os tecidos orgânicos (Golley et al., 1978).

Segundo Figueiredo Filho et al. (2005), vários fatores abióticos e bióticos como tipo de vegetação, precipitação, altitude, temperatura, latitude, luminosidade, relevo, deciduosidade, estágio sucessional, disponibilidade hídrica e características do solo afetam a produção de serapilheira. Dependendo das características de cada ecossistema, um determinado fator pode prevalecer sobre os demais.

Entre os sistemas avaliados, o sistema agrissilvipastoril com eucalipto, aos 11 anos, em MG apresentou a maior estocagem pela serapilheira com 10,89 $\mathrm{tC} \mathrm{ha}^{-1}$, seguido pelo sistema silvipatoril com Pinus elliottii (400 árv. ha $^{-1}$ ), aos 30 anos, no Estado de SP, com 5,81 t C ha-1.

Esses valores foram inferiores ao encontrado por Reis et al. (1994), em plantios de eucalipto no Estado do Espírito Santo, aos 7 anos, em que a estocagem foi de $12,38 \mathrm{t} \mathrm{Cha}^{-1}$, mas próximos ao observado por Paixão (2004), na serapilheira em um povoamento de eucalipto, aos 6 anos, no Município de Viçosa, $\mathrm{MG}\left(8,72\right.$ t C ha $\left.{ }^{-1}\right)$. Solos

A quantidade de carbono orgânico no solo está relacionada com a entrada de material orgânico, por meio da senescência de certos componentes da biomassa acima e abaixo do solo, queda das folhas, resíduos da exploração e animais mortos, com suas respectivas taxas de decomposição (Balbinot et al., 2003). Segundo Lehmann \& Zech (1998), de $20 \%$ a $50 \%$ da renovação das raízes e de $10 \%$ a $20 \%$ da serapilheira é transformada em matéria orgânica do solo.

Nos sistemas agroflorestais avaliados, a estocagem de carbono no solo variou entre 29,93 $\mathrm{t} \mathrm{ha}^{-1}$ e 97,24 t ha $^{-1}$. Esses valores foram inferiores aos encontados por Corazza et al. (1999), que estudaram a estocagem de carbono em uma área de vegetação típica de cerrado e outra com plantio de eucalipto com 12 anos (Tabela 3 ).

Tabela 3. Conteúdo de carbono nas diferentes camadas no solo.

\begin{tabular}{ccc}
\hline \multirow{2}{*}{ Camada de solo $(\mathbf{c m})$} & \multicolumn{2}{c}{$\begin{array}{c}\text { Conteúdo de carbono } \\
\left(\mathbf{t} \mathbf{~ h a}^{-1}\right)\end{array}$} \\
\cline { 2 - 3 } & Cerrado & Eucalipto \\
\hline $0-20$ & 39,77 & 44,87 \\
$20-40$ & 30,09 & 33,50 \\
$40-100$ & 63,72 & 69,81 \\
\hline Total & 133,59 & 148,19 \\
\hline
\end{tabular}

Fonte: Corazza et al. (1999).
Pulrolnik et al. (2009) verificou que o cerrado sensu stricto, em Itamarandiba, MG, apresentou uma estocagem de carbono orgânico total inferior a um plantio de eucalipto, aos 20 anos, e também a uma pastagem (Tabela 4). Isso se deve, provavelmente, ao sistema radicular de ciclagem rápida da pastagem e à maior produção de serapilheira no eucalipto.

Tabela 4. Estoques de carbono orgânico total nas diversas camadas de solo sob Cerrado, pastagem e eucalipto.

\begin{tabular}{cccc}
\hline \multirow{2}{*}{$\begin{array}{c}\text { Camada de } \\
\text { solo }(\mathbf{c m})\end{array}$} & \multicolumn{3}{c}{ Conteúdo de carbono $\left(\mathbf{t} \mathbf{h a}^{-1}\right)$} \\
\cline { 2 - 4 } & Cerrado & Pastagem & Eucalipto \\
\hline $0-10$ & 22,39 & 23,88 & 23,57 \\
$10-20$ & 21,18 & 23,83 & 22,26 \\
$20-40$ & 30,69 & 34,45 & 35,65 \\
$40-60$ & 22,63 & 26,40 & 26,93 \\
$60-100$ & 35,80 & 41,93 & 44,04 \\
\hline Total & 132,69 & 150,49 & 152,45 \\
\hline
\end{tabular}

Fonte: Pulrolnik et al. (2009)

\section{Considerações finais}

Os sistemas agroflorestais contribuem para captura de $\mathrm{CO}_{2}$ atmosférico e sua estocagem na superfície terrestre. Esse processo fortalece a importância dos SAFs na mitigação das mudanças climáticas.

A estocagem de carbono nos SAFs variou em função do arranjo implantado e da idade do sistema, sendo que o sistema agroflorestal regenerativo análogo apresentou maior potencial, alcançando $11,19 \mathrm{t} \mathrm{C} \mathrm{ha}^{-1}$.

A maior capacidade de estocagem de carbono nos sistema agroflorestal regenerativo análogo ocorre em função do maior número de espécies arbóreas e/ou arbustivas existentes por unidade de área.

Os sistemas agroflorestais são estratégicos na estocagem de carbono e mitigação da emissão de GEE. Estes sistemas devem ser incentivados no Plano Nacional sobre Mudanças do Clima, de forma a tornar as práticas agropecuárias ambientalmente mais sustentáveis.

\section{Referências}

ALBRECHT, A.; KANDJI, S. T. Carbon sequestration in tropical agroforestry systems. Agriculture, Ecosystems and Environment, Amsterdam, NL, v. 99, n. 1, p. 15-27, 2003.

BALBINOT, R.; SCHUMACHER, M. V.; WATZLAWICK, L. F.; SANQUETTA, C. R. Inventário do carbono orgânico em um plantio de Pinus taeda aos 5 anos de idade no Rio Grande do Sul. Revista Ciências Exatas e Naturais, Guarapuava, v. 5, n. 1, 2003. 
BERTALOT, M. J. A. Cultura do milho (Zea mays L.) em sucessão com aveia preta (Avena strigosa Schreb.) em áreas sob manejo agroflorestal em aléias com Leucaena diversifolia. 2003. $88 \mathrm{f}$. Tese (Doutorado em Agronomia) - Universidade Estadual Paulista, Botucatu.

BOLFE, E. L.; FERREIRA, M. C.; BATISTELLA, M. Biomassa epígea e estoque de carbono de agroflorestas em Tomé-Açu, PA. Revista Brasileira de Agroecologia, Espírito Santo, v. 4, n. 2, p. 2171-2175, 2009.

BOWEN, G. D. Roots as a component of tree productivity. In: CANNELL, M. G. R.; JACKSON, J. E. (Ed.). Attributes of trees as crop plants. Huntingdon, UK: Institue of Terrestrial Ecology, 1985. p. 303-315.

BRANCHER, T. Estoque e ciclagem de carbono de sistemas agroflorestais em Tomé-Açu, Amazônia Oriental. 2010. 58 f. Dissertação (Mestrado em Ciências Ambientais) - Universidade Federal do Pará, Belém, PA.

BRASIL. Ministério da Agricultura, Pecuária e Abastecimento. Plano setorial de mitigação e de adaptação às mudanças climáticas para a consolidação de uma economia de baixa emissão de carbono na agricultura. Brasília, DF, 2011. 75 p. Versão preliminar.

BRASIL. Ministério da Ciência, Tecnologia e Inovação. Segunda comunicação inicial do Brasil: parte II: inventário de emissões e remoções antrópicas de gases de efeito estufa não controlados pelo Protocolo de Montreal. Brasília, DF, 2010. 102 p.

BRASSARD, B. W.; CHEN, H. Y. N.; BERGERON, Y.; PARÉ, D. Coarse root biomass allometric equations for Abies balsamea, Picea mariana, Pinus banksiana, and Populus tremuloides in the boreal forest of Ontario, Canada. Biomass and Bioenergy, Oxford, v. 35, n. 10, p. 4189-4196, 2011.

BROWN, S. Estimating biomass and biomass change of tropical forests: a primer. Rome: FAO, 1997. 55 p.

CAIRNS, M. A.; BROWN, S.; HELMER, E. H.; BAUMGARDNER G. A. Root biomass allocation in the world's upland forests. Oecologia, v. 111, n. 1, p. 1-11, 1997.

CALVI, G. P.; PEREIRA, M. G.; ESPÍNDULA JÚNIOR, A. Produção de serapilheira e aporte de nutrientes em áreas de Floresta Atlântica em Santa Maria de Jetibá, ES. Ciência Florestal, Santa Maria, RS, v. 19, n. 2, 2009.

COLTRI, P. P.; LAZARIM, C.; DIAS, R.; ZULLO JUNIOR, J.; PINTO, H. S. Estoque de carbono em sistemas cafeeiros a pleno sol e cultivado com macadâmia no sul de Minas Gerais, Brasil. In: SIMPÓSIO DE PESQUISA DOS CAFÉS DO BRASIL, 7., 2011, Araxá. Anais...Brasília, DF: Embrapa Café, 2011.

CORAZZA, E. J.; SILVA J. E.; RESCK, D. V. S.; GOMES, A. C. Comportamento de diferentes sistemas de manejo como fonte ou depósito de carbono em relação à vegetação de cerrado. Revista Brasileira de Ciência do Solo, Campinas, v. 23, n. 2, p. 425-432, 1999.

COTTA, M. K. Quantificação de biomassa e análise econômica do consórcio seringueira-cacau para geração de créditos de carbono. 2005. 89 f. Dissertação (Mestrado em Ciência Florestal) - Universidade Federal de Viçosa, Viçosa, MG.
DaMATTA, F. M. Fisiologia do cafeeiro em sistemas arborizados. In: MATSuMOTO, S. N. (Org.). Arborização de Cafezais no Brasil. Vitória da Conquista: Edições UESB, 2004. p. 23-41.

DECONTO, J. G. (Coord.). Aquecimento global e a nova geografia da produção agrícola no Brasil. Campinas: Embrapa Informática Agropecuária, 2008. 82 p.

DUBOIS, J. C. L.; VIANA, V. M.; ANDERSON A. B. Manual agroflorestal para a Amazônia. Rio de Janeiro: REBRAF, 1996. v. $1.228 \mathrm{p}$.

ENGEL, V. L. Introdução aos sistemas agroflorestais. Botucatu: FEPAF, 1999. $70 \mathrm{p}$.

ESPIG, S. A.; FREIRE, F. J.; MARANGON, L. C.; FERREIRA, R. L C.; FREIRE, M. B. G. dos S.; ESPIG, D. B. Sazonalidade, composição e aporte de nutrientes da serapilheira em fragmento de Mata Atlântica. Revista Árvore, Viçosa, MG, v. 33, n. 5, p. 949956, 2009.

FIGUEIREDO FILHO, A.; SERPE, E. L.; BECKER, M.; SANTOS, D. F. Produção estacional de serapilheira em uma floresta Ombrófila Mista na Floresta Nacional de Irati (PR). Ambiência: Revista do Centro de Ciências Agrárias e Ambientais, Guarapuava, v. 1, n. 2 , p. 257-269, 2005.

FROUFE, L. C. M.; RACHWAL, M. F. G.; SEOANE, C. E. S. Potencial de sistemas agroflorestais multiestrata para sequestro de carbono em áreas de ocorrência de Floresta Atlântica. Pesquisa Florestal Brasileira, Colombo, v. 31, n. 66, p. 143-154, 2011. DOI: 10.4336/2011.pfb.31.66.143

GOLLEY, F. B.; McGINNIS, J. T.; CLEMENTS, R. G.; CHILD, G. I.; DUEVER, M. J. Ciclagem de minerais em um ecossistema de floresta tropical úmida. São Paulo: EDUSP,1978. 256 p.

GÖTSCH, E. Break-through in agriculture. Rio de Janeiro: ASPTA, 1995. 22 p.

GUTMANIS, D. Estoque de carbono e dinâmica ecofisiológica em sistemas silvipastoris. 2004. 142 f. Tese (Doutorado em Ciências Biológicas) - Universidade Estadual Paulista, Rio Claro, SP.

LEHMANN, J.; ZECH, W. Fine root turnover of irrigated hedgerow intercropping in Northern Kenya. Plant and Soil, The Hague, v. 198, p. 19-31, 1998.

LOBÃO, D. E.; SETENTA W. C.; VALle, R. R. Sistema agrossilvicultural cacaueiro: modelo de agricultura sustentável. Agrossilvicultura, Viçosa, MG, v. 1, n. 2, p. 163-173, 2004.

MAFRA, A. L.; MIKLÓS, A. A. W.;VOCURCA, H. L.; HARKALY, A. H.; MENDOZA, E. Produção de fitomassa e atributos químicos do solo sob cultivo em aléias e sob vegetação nativa de cerrado. Revista Brasileira de Ciência do Solo, Campinas, v. 22, p. 43-48, 1998.

MELLO, S. L. M.; GONÇALVES, J. L. M. Equações para estimar a biomassa da parte aérea e do sistema radicular em povoamentos de Eucalyptus grandis em sítios com produtividades distintas. Revista Árvore, Viçosa, MG, v. 32, n. 1, p. 101-111, 2008.

MONTAGNINI, F.; NAIR, P. K. R. Carbon sequestration: an underexploited environmental benefit of agroforestry system. Agroforestry Systems, Dordrecht, NL, v. 61, n. 1/3, p. 281-298, 2004. 
MOSQUERA-LOSADA, M. R.; FREESE, D.; RIGUEIRORODRÍGUEZ, A. Carbon sequestration in European Agroforestry Systems. In: KUMAR, B. M.; NAIR, P. K. R. (Ed.). Carbon sequestration potential of agroforestry systems: opportunities and challenges. London: New York: Springer, 2011. p. 43-59.

MÜLlER, M. D.; FERNANDES, E. N.; CASTRO, C. R. T.; PACIULLO, D. S. C.; ALVES, F. F. Estimativa de acúmulo de biomassa e carbono em sistema agrossilvipastoril na Zona da Mata Mineira. Pesquisa Florestal Brasileira, Colombo, n. 60, p. 11-17, 2009. Edição especial.

NAIR, P. K. R. An introduction to agroforestry. Dordrecht, NL, NL: Kluwer Academic Publishers, 1993. 499 p.

NAIR, P. K. R. Classification of agroforestry systems. Agroforestry Systems, Dordrecht, NL, v. 3, n. 2, p. 97-128, 1985.

NAIR, P. K. R.; TONUCCI, R. G.; GARCIA, R.; NAIR, V. D. Silvopasture and carbon sequestration with special reference to the Brazilian savanna (Cerrado). In: KUMAR, B. M.; NAIR, P. K. R. (Ed.). Carbon sequestration potential of agroforestry systems: opportunities and challenges. London: New York: Springer, 2011. p.145-162.

OELBERMANN, M.; VORONEY, R. P.; GORDON, A. M. Carbon sequestration in tropical and temperate agroforestry systems: a review with examples from Costa Rica and southern Canada. Agriculture, Ecosystems and Environment, Amsterdam, NL, v. 104, p. 359-377, 2004.

OLIVEIRA, E. B. de; RIBASKI, J.; ZANETTI, É. A.; PENTEADO JÚNIOR, J. F. Produção, carbono e rentabilidade econômica de Pinus elliottii e Eucalyptus grandis em sistemas silvipastoris no Sul do Brasil. Pesquisa Florestal Brasileira, Colombo, n. 57, p. 45-56, 2008.

OSTONEN, I.; LÕHMUS, K.; PAJUSTE, K. Fine root biomass, production and its proportion of NPP in a fertile middle-aged Norway spruce forest: Comparison of soil core and ingrowth core methods. Forest Ecology and Management, Amsterdam, NL, v. 212, n. 1-3, p. 264-277, 2005.

PAIXÃO, F. A. Quantificação do estoque de carbono e avaliação econômica de alternativas de uso de um povoamento de eucalipto. 2004. 50 f. Dissertação (Mestrado em Ciência Florestal) - Universidade Federal de Viçosa, Viçosa, MG.

PENNAM, J.; GYTARSKY, M.; HIRAISHI, T.; KRUG, T.; KRUGER, D.; PIPATTI, R.; BUENDIA, L.; MIWA, K.; NGARA, T.; TANABE, K.; WAGNER, F. (Ed.). Good practice guidance for land use, land use change and forestry. Prepared by the National Greenhouse Gas Inventories: programme. Hayama, Kanagawa: Institute for Global Environmental Strategies for the IPCC; The Intergovernmental Panel on Climate Change (IPCC), 2003.
PIASENTIN, F. A.; SAITO, C. H. Caracterização do cultivo de cacau na região econômica litoral sul, sudeste da Bahia. Estudo \& Debate, Lageado, v. 19, n. 2, p. 63-80, 2012.

PORFÍRIO-DA-SILVA, V. Arborização de pastagens: I. Procedimentos para introdução de árvores em pastagens. Colombo: Embrapa Florestas, 2006. 8 p. (Embrapa Florestas. Comunicado técnico, 155).

PULROLNIK, K.; BARROS, N. F. de; SILVA, I. R.; NOVAIS, R. F.; BRANDANI, C. B. Estoques de carbono e nitrogênio em frações lábeis e estáveis da matéria orgânica de solos sob eucalipto, pastagem e cerrado no Vale do Jequitinhonha - MG. Revista Brasileira de Ciência do Solo, Campinas, v. 33, n. 5, p. 1125-1136, 2009.

REIS, M. das G. F. REIS, G. G; VALENTE, O. F.; FERNANDES, H. A. C. Seqüestro e armazenamento de carbono em florestas nativas e plantadas dos estados de Minas Gerais e Espírito Santo. In: SEMINÁRIO EMISSÃO X SEQUESTRO DE CO $\mathrm{C}_{2}, 1 ., 1994$, Rio de Janeiro. Anais... Rio de Janeiro: Companhia Vale do Rio Doce, 1994. p. $155-195$.

RODRIGUES, V. G. S.; CASTILLA, C.; COSTA, R. C. da; PALM, C. Estoque de carbono em sistema agroflorestal com café em Rondônia - Brasil. In: SIMPÓSIO DE PESQUISA DOS CAFÉS DO BRASIL, 1., 2000, Poços de Caldas. Resumos Expandidos. [Poços de Caldas: s.n., 2000].

SAMBUICHI, R. H. R. Estrutura e dinâmica do componente arbóreo em área de cabruca na região cacaueira do sul da Bahia. Acta Botanica Brasilica, Porto Alegre, v. 20, n. 4, p. 943-954, 2006.

SANTOS, S. R. M.; MIRANDA, I. S.; TOURINHO, M. M. Estimativa de biomassa de sistemas agroflorestais das varzeas do rio Juba, Cameta, PA. Acta Amazonica, Manaus, v. 34, n. 1, p. 1-8, 2004.

SCHUMACHER, M. V.; HOPPE, J. M. A complexidade dos ecossistemas. Porto Alegre: Pallotti, 1997. 50 p.

SCHUMACHER, M. V.; WITSCHORECK, R. Inventário de carbono em povoamentos de Eucalyptusssp nas propriedades fumageiras do sul do Brasil: "um estudo de caso". In: SIMPÓSIO LATINO AMERICANO SOBRE FIXAÇÃO DE CARBONO, 2004, Curitiba. Fixação de carbono: atualidades, projetos e pesquisas. Curitiba: UFPR; Instituto Ecoplan, 2004. Part. 7. p. 111-124.

TSUKAMOTO FILHO, A. A. Fixação de carbono em um sistema agroflorestal com eucalipto na região do Cerrado de Minas Gerais. 2003. 98 f. Tese (Doutorado em Ciência Florestal) Universidade Federal de Viçosa, Viçosa, MG.

VIVAN, J. L. Agricultura e Florestas: princípios de uma interação vital. Guaíba, RS: Agropecuária, 1998. 207 p.

WINROCK INTERNATIONAL. A guide to monitoring carbon storage in forestry and agroforetry projects. California: California University, 1997. 87 p. 